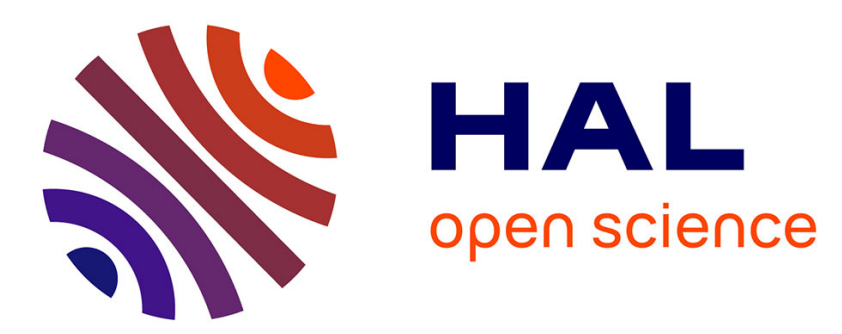

\title{
Enhanced transmission through subwavelength metallic coaxial apertures by excitation of the TEM mode F.I. Baida
}

\section{To cite this version:}

F.I. Baida. Enhanced transmission through subwavelength metallic coaxial apertures by excitation of the TEM mode. Applied Physics B - Laser and Optics, 2007, 89 (2-3), pp.145-149. 10.1007/s00340007-2787-3 . hal-00250026

\section{HAL Id: hal-00250026 \\ https://hal.science/hal-00250026}

Submitted on 6 May 2021

HAL is a multi-disciplinary open access archive for the deposit and dissemination of scientific research documents, whether they are published or not. The documents may come from teaching and research institutions in France or abroad, or from public or private research centers.
L'archive ouverte pluridisciplinaire HAL, est destinée au dépôt et à la diffusion de documents scientifiques de niveau recherche, publiés ou non, émanant des établissements d'enseignement et de recherche français ou étrangers, des laboratoires publics ou privés. 


\section{Enhanced transmission through subwavelength metallic coaxial apertures by excitation of the TEM mode}

\author{
F.I. BAIDA
}

Institut FEMTO-ST, UMR 6174 CNRS, Université de Franche-Comté, 25030 Besançon Cedex, France

\begin{abstract}
We present here the first theoretical demonstration of enhanced transmission (up to 90\%) through annular aperture arrays engraved into opaque metallic plates thanks to the excitation of the TEM guided mode inside each coaxial cavity. The generation of this peculiar mode is obtained, first, by illuminating the structure under oblique incidence and, second, by considering a TM polarization. Analytical demonstration is performed to confirm the involvement of these two conditions for the emergence of this guided mode. The originality of this configuration comes, first, from the fact that the TEM mode has no cut-off wavelength and, second, from the fact that the transmission peak position is independent of the angle of incidence.
\end{abstract}

Light interaction with metallic plates perforated with subwavelength apertures is now a wide area of research. This infatuation is mainly due to the recent technological progress in the domain of nanofabrication allowing prospective manufacturing of very large periodic structures with both high filling factors and high aspect ratios. Based on this, new generations of photonic crystals or other collective structures are born, promoting broad functionalities and opening up the path to a novel kind of material exhibiting extraordinary physical properties that were previously unknown [1] or insignificant [2]. Thereby, the exaltation of linear $[3,4]$ or nonlinear $[5,6]$ responses of the matter can directly be obtained by a specific nanostructuring of the material used. Other phenomena such as the beaming of the light $[7,8]$, invisible bodies $[9,10]$ or perfect lenses $[11,12]$ are now real technological challenges that could be achieved thanks to the mastering of the light by controlling the electromagnetic field at the nanometre scale [13]. In all cases, the explanation of the sustaining physical phenomenon plays a fundamental role, in the long term, for a potential commercialisation of the products. In this context, frequency-selective surfaces (FSSs) [14] exhibiting original properties were recently proposed in the optical domain $[15,16]$ in order to extend their well-known potentialities to the visible range. The basic idea consists then in the use of such a structure to master the light emerging - reflected or transmitted - from it. Special properties were latterly demonstrated in the visible and in the infrared domains $[16,17]$.

This paper deals with the study of a FSS structure based on periodically arranged subwavelength coaxial apertures engraved into metallic layers. Let us first recall why we chose this structure. The first idea that brought Baida and Van Labeke [4] to propose this kind of structure to enhance the light transmission is linked to the fact that a coaxial waveguide made in a perfect conductor has a guided mode without cut-off, i.e. the TEM mode. But, at normal in- cidence, a linearly polarized plane wave permits us to excite the $\mathrm{TE}_{11}$ mode [18] because it is the only mode that can match the incident linear polarization (taking into consideration the symmetry of the waveguide). The TEM mode, which is independent of the azimuthal angle, cannot be excited in this configuration. In fact, at normal incidence, it can only be generated if we consider radially polarized incident beams. However, such beams, which are currently obtained by selecting the $\mathrm{TM}_{01}$ mode of an optical fibre [19], are spatially limited and allow the illumination of a small number of patterns. Thus, it is of great interest to determine the conditions to fulfil in order to excite the TEM mode using a linearly polarized plane wave. If we succeed in doing this, then we can tailor a novel configuration based on a vast periodic structure allowing high transmission at any desired value of the wavelength.

Thus, we will demonstrate the possible generation and the propagation of this TEM mode inside each annular aperture when illuminating the whole structure by a linearly polarized incident plane wave under oblique incidence. This property will be analytically demonstrated by expressing the EM field of the incident plane wave through its angular spectrum. For a perfect metallic coaxial waveguide, the TEM mode is completely determined by only two components of the EM field, i.e. $E_{\mathrm{r}}$ and $H_{\varphi}$. This means that the electric field is radial and the magnetic field is azimuthal. The other four components are zero. In addition, these two components do not depend on the azimuthal angle. The basic idea is then: if we want to excite this mode, we should 
illuminate the structure with an EM field having simultaneously non-zero values for $E_{\mathrm{r}}$ and $H_{\varphi}$ components.

To analytically determine the consequences of this condition, let us express these two components in the case of a linear polarization. Figure 1a shows the geometrical parameters that are necessary to define the incident EM field. The angle of incidence is set to $\theta$ and we fix the wavevector to be parallel to the $(x, z)$ plane. This can be obtained by rotational symmetry without any restriction on the illumination.

In the Cartesian coordinate system, the wavevector $\boldsymbol{k}$ can be expressed by

$\boldsymbol{k}=k\left(\sin \theta \boldsymbol{e}_{x}+\cos \theta \boldsymbol{e}_{z}\right)$,

where $k$ is the modulus of the wavevector.

In the cylindrical coordinate system, we obtain

$$
\begin{aligned}
\boldsymbol{k}= & k\left(\sin \theta \cos \varphi \boldsymbol{e}_{\mathrm{r}}-\sin \theta \sin \varphi \boldsymbol{e}_{\varphi}\right. \\
& \left.+\cos \theta \boldsymbol{e}_{z}\right) .
\end{aligned}
$$

In order to determine the electromagnetic field components in cylindrical coordinates, we consider a generally polarized plane wave having both TE and TM components. The divergence of the electric field allows us to express these two components by

$$
\begin{aligned}
& \boldsymbol{E}_{\mathrm{TE}}=E_{\mathrm{TE}} \frac{\boldsymbol{k} \wedge \boldsymbol{e}_{z}}{\left\|k_{\|}\right\|}, \\
& \boldsymbol{E}_{\mathrm{TM}}=E_{\mathrm{TM}} \frac{\boldsymbol{E}_{\mathrm{TE}} \wedge \boldsymbol{k}}{\left\|\boldsymbol{E}_{\mathrm{TE}} \wedge \boldsymbol{k}\right\|} .
\end{aligned}
$$

By inserting (1) into these last two equations, one obtains

$$
\begin{aligned}
\boldsymbol{E}_{\mathrm{TE}}= & E_{\mathrm{TE}}\left\{-\sin \varphi \boldsymbol{e}_{\mathrm{r}}+\cos \varphi \boldsymbol{e}_{\varphi}\right\} \\
\boldsymbol{E}_{\mathrm{TM}}= & E_{\mathrm{TM}}\left\{\cos \theta \cos \varphi \boldsymbol{e}_{\mathrm{r}}\right. \\
& \left.+\cos \theta \sin \varphi \boldsymbol{e}_{\varphi}+\sin \theta \cos 2 \varphi \boldsymbol{e}_{z}\right\} .
\end{aligned}
$$

In addition, we have to express the spatial phase in the same cylindrical coordinate system. This leads to

$$
\begin{aligned}
\boldsymbol{k} \cdot \boldsymbol{R} & =\boldsymbol{k}_{\|} \cdot \boldsymbol{r}+k_{z} z \\
& =k r \sin \theta \cos \varphi+k z \cos \theta .
\end{aligned}
$$

Finally, when expressed in the cylindrical coordinates, the three components of the electric field become

$$
\begin{aligned}
E_{\mathrm{r}}(r, \varphi, z, t)= & A\left\{-E_{\mathrm{TE}} \sin \varphi\right. \\
& \left.+E_{\mathrm{TM}} \cos \theta \cos \varphi\right\}, \\
E_{\varphi}(r, \varphi, z, t)= & A\left\{E_{\mathrm{TE}} \cos \varphi\right. \\
& \left.+E_{\mathrm{TM}} \cos \theta \sin \varphi\right\}, \\
E_{z}(r, \varphi, z, t)= & A E_{\mathrm{TM}} \sin \theta \cos 2 \varphi,
\end{aligned}
$$

where $A=\mathrm{e}^{-\mathrm{i}(\omega t-k r \sin \theta \cos \varphi-k z \cos \theta)}$.

Similar equations can be found for the magnetic field via the MaxwellFaraday equation. Let us give only the $\varphi$ component:

$$
\begin{aligned}
H_{\varphi}(r, \varphi, z, t)=- & B\left\{E_{\mathrm{TE}} \cos \theta \cos \varphi\right. \\
+ & E_{\mathrm{TM}}\left(\cos ^{2} \theta \cos \varphi\right. \\
& \left.\left.+\sin ^{2} \varphi \cos 2 \varphi\right)\right\}
\end{aligned}
$$

where

$B=\frac{-k}{\mu \omega} \times \mathrm{e}^{-\mathrm{i}(\omega t-k r \sin \theta \cos \varphi-k z \cos \theta)}$.

Furthermore, these components can be expanded by their Fourier sine and cosine series. In a general way, we can

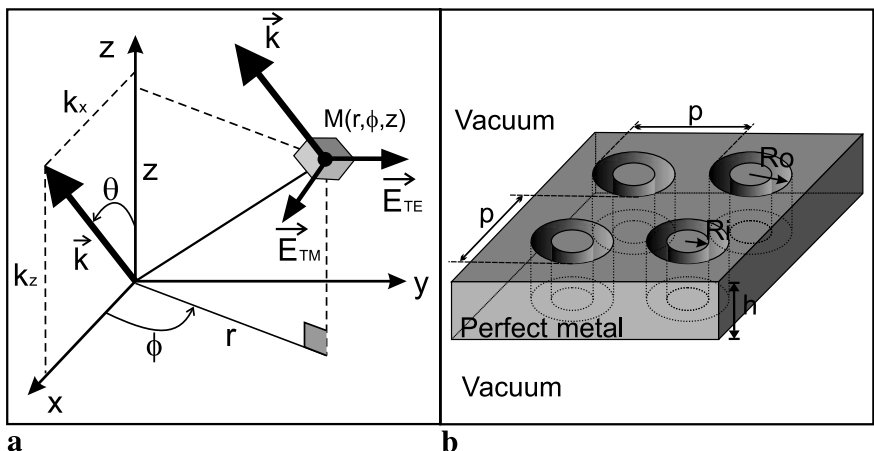

FIGURE 1 (a) The geometrical parameters used to define the wavevector direction in both cylindrical and Cartesian coordinate systems. (b) The schema of the studied AAA structure

write them through a series of exponential terms. For example, the radial component of the electric field can be expressed by

$$
\begin{aligned}
E_{\mathrm{r}}(r, \varphi, z, t)= & E_{\mathrm{r}}^{0}(r, z, t) \\
& +\sum_{m=1}^{\infty} E_{\mathrm{r}}^{m}(r, z, t) \mathrm{e}^{\mathrm{i} m \varphi},
\end{aligned}
$$

with

$$
\begin{aligned}
& E_{\mathrm{r}}^{0}(r, z, t)=\frac{1}{2 \pi} \int_{0}^{2 \pi} E_{\mathrm{r}}(r, \varphi, z, t) \mathrm{d} \varphi, \\
& E_{\mathrm{r}}^{m}(r, z, t)=\frac{1}{\pi} \int_{0}^{2 \pi} E_{\mathrm{r}}(r, \varphi, z, t) \mathrm{e}^{\mathrm{i} m \varphi} \mathrm{d} \varphi .
\end{aligned}
$$

As mentioned above, the TEM mode has no dependence on $\varphi$. Thus, it holds only components for $m=0$. The $E_{\mathrm{r}}^{0}$ component is evaluated from (4) and (7) as for the two other components of the electric field, while the $H_{\varphi}^{0}$ one is calculated from (5) and (7).

Because of paper length, we will only present results for the $E_{\mathrm{r}}^{0}$ and the $H_{\varphi}^{0}$ components. After some algebra, one obtains

$$
\begin{aligned}
& E_{\mathrm{r}}^{0}(r, z, t)=C \cos \theta E_{\mathrm{TM}} J_{1}(k r \sin \theta), \\
& H_{\varphi}^{0}(r, z, t)=\frac{-C k}{\mu \omega} E_{\mathrm{TM}} \\
& \quad \times\left\{\left(\frac{3}{4} \cos 2 \theta+\frac{1}{4}\right) J_{1}(k r \sin \theta)\right. \\
& \left.\quad+\frac{\sin ^{2} \theta}{2} J_{3}(k r \sin \theta)\right\},
\end{aligned}
$$

with $C=\mathrm{ie}^{-\mathrm{i}(\omega t-k z \cos \theta)}$.

It is clearly shown from (8) that both the $E_{\mathrm{r}}^{0}$ and $H_{\varphi}^{0}$ components are non-zero only if the incident electric field has a TM component. This condition is necessary but insufficient because the Bessel functions ( $J_{1}$ and $J_{3}$ ) should also be non-zero whatever is the value of $r$. This second constraint is simply verified when the structure is illuminated under off-normal incidence $(\theta \neq 0)$. Thus, the TEM mode can only be excited in TM polarization under offnormal incidence.

To numerically confirm this finding, we use a finite difference time domain (FDTD) algorithm that was recently developed by us [20] in order to handle the 
study of periodic structures illuminated in oblique incidence.

For this goal, we consider a structure based on an annular aperture array (AAA) made in a perfect conductor (see Fig. 1b). Otherwise, we consider a self-suspended configuration where the metallic plate is surrounded by vacuum in order to have a simple interpretation of the obtained results.

In order to take away the Rayleigh anomaly from the transmission peak that corresponds to the excitation and the propagation of the $\mathrm{TE}_{11}$ guided mode, we have adjusted the geometrical parameters of the annular aperture by setting the inner radius to $R_{\mathrm{i}}=p / 4$ and the outer one to $R_{\mathrm{o}}=p / 3, p$ being the period of the AAA structure. In fact, this last mode (the $\mathrm{TE}_{11}$ one) is at the origin of the enhanced transmission through such structures when illuminated under normal incidence [18]. It has a cut-off wavelength at $\lambda_{\mathrm{c}}^{\mathrm{TE}_{11}} \simeq \pi\left(R_{\mathrm{o}}+R_{\mathrm{i}}\right)$. In our case, this value is equal to $\lambda_{\mathrm{c}}^{\mathrm{TE}_{11}} \simeq$ $(1.83 \pm 0.2) p$. This uncertainty is due to the spatial meshing of the structure (imprecision in the values of $R_{\mathrm{i}}$ and $R_{\mathrm{O}}$ ). Let us note also that because of the spatial Cartesian meshing, a staircase effect can appear leading to a small red shift of this cut-off wavelength.

The transmission spectra of a $2 p$ thick AAA structure are presented in Fig. 2 for different values of the angle of incidence and in the case of a TE polarization. As was demonstrated in [16], the structure is angle-independent, i.e. the position and width of the transmission peaks do not depend on the angle of incidence until the occurrence of additional homogeneous diffracted orders. This can be seen in Fig. 2 through the change of the amplitude of the first peak (the left-hand one) for $\theta=40^{\circ}$. Contrariwise, additional transmission peaks appear in the case of TM polarization (see Fig. 3) even beyond $\lambda_{\mathrm{c}}^{\mathrm{TE}_{11}}$. The amplitude of these last peaks increases with $\theta$ and their positions remain independent of the angle of incidence. In fact, the positions of the TEM peaks depend only on the length of each annular cavity, i.e. on the metal thickness.

The finite length of the annular waveguides, which can be considered as opened Fabry-Pérot cavities, plays the main role in the TEM peaks' positions [21]. When the TEM mode is

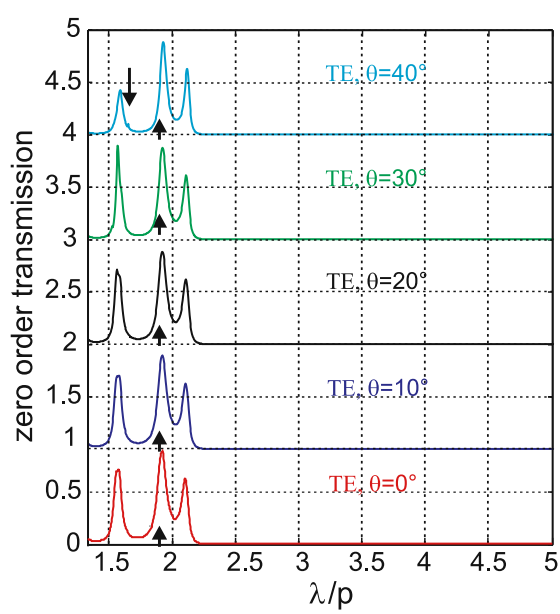

FIGURE 2 Transmission spectra of the AAA structure for five different values of the angle of incidence in the case of a TE linearly polarized incident plane wave. Spectra are shifted vertically by 1 for clarity. As was demonstrated in [16], the spectral response of such a structure is almost independent of the angle of incidence for wavelengths larger than the one corresponding to the occurrence of the Rayleigh anomaly (see the down-arrow located at $\lambda / p \simeq 1.64$ for $\theta=40^{\circ}$ ). Note that the up-arrows indicate the position of the cut-off of the $\mathrm{TE}_{11}$ guided mode

excited, the AAA structure becomes equivalent to a $2 \mathrm{D}$ metallic lamellar grating (slits), for which resonances also occur under normal incidence.

For a coaxial waveguide made in a perfect conductor, the effective index of the TEM mode is equal to 1 . Thus, the occurrence of a TEM transmission peak is fully governed by a phase-matching condition:

$\lambda_{\mathrm{TEM}}=\frac{2 \pi h}{l \pi-\psi_{\mathrm{r}}}$,

where $\lambda_{\text {TEM }}$ is the wavelength relative to the TEM peak, $l$ is a non-zero positive integer and $\psi_{\mathrm{r}}$ is the phase change occurring at the reflection of the guided mode at the end of the coaxial aperture. Its value can then be deduced from the spectra of Fig. 3 via (9).

To confirm the excitation of the TEM mode, we present in Fig. $4 \mathrm{a}$ and $\mathrm{b}$ the radial $E_{\mathrm{r}}(x, y)$ and the axial $E_{z}(x, y)$ electric field components. They are calculated inside the annular cavities at $100 \mathrm{~nm}$ from the exit side of the structure. The value of the wavelength corresponds to the largest $-\lambda$ TEM peak when the structure is illuminated under $\theta=40^{\circ}$ (see Fig. 3). As expected, the radial component of the electric field is independent of the azimuthal angle

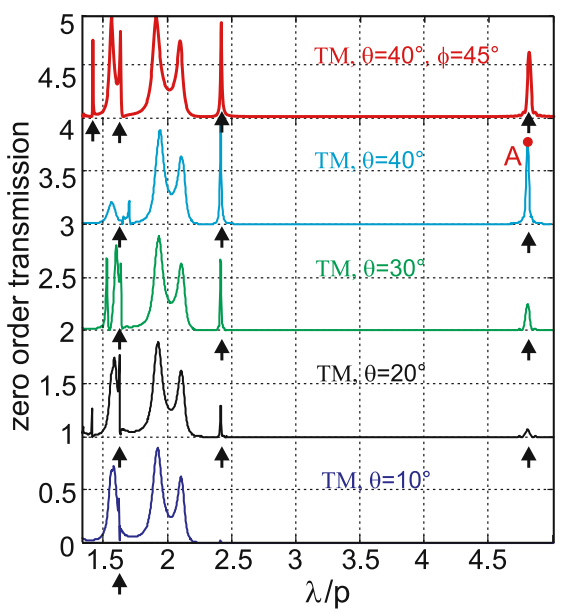

FIGURE 3 Transmission spectra of the AAA structure for four different values of the angle of incidence in the case of a TM linearly polarized incident plane wave. In addition, we present, for the upper curve, the spectral response in the case of an incident wavevector directed toward the unit vector $\boldsymbol{u}=\left((1 / \sqrt{2}) \sin 40^{\circ},(1 / \sqrt{2}) \sin 40^{\circ}\right.$, $\left.\cos 40^{\circ}\right)$. Spectra are shifted vertically by 1 for clarity. As is expected, the spectral responses present extra peaks (pointed out by up-arrows) that are linked to the excitation and the propagation of the TEM guided mode inside each coaxial aperture

while the two other components are almost zero.

In summary, we have presented a complete and comprehensive study of the enhanced transmission through coaxial apertures thanks to the excitation of TEM guided modes. The corresponding transmission peaks are very narrow $(\lambda / \Delta \lambda \simeq 250)$ compared to the one located at the cut-off of the $\mathrm{TE}_{11}$ mode. This property is of great importance for spectral filtering. In addition, the peak position can be tuned by simply modifying the refractive index of the medium placed inside the cavities. Even if the TEM mode does not have a cut-off wavelength, there exists an upper limit imposed by the phase-matching condition exactly as for a conventional Fabry-Pérot cavity. For microwave and terahertz domains, this limit is not really a problem because it is easy to build structures with very high aspect ratios. Unfortunately, for the optical range, the fabrication of nanometric high aspect ratio structures becomes a very difficult task. Furthermore, in the visible domain, noble metals cannot be considered as perfect conductors and they present losses that prohibit the use of thick metallic plates [4]. In fact, the real nature of no- 


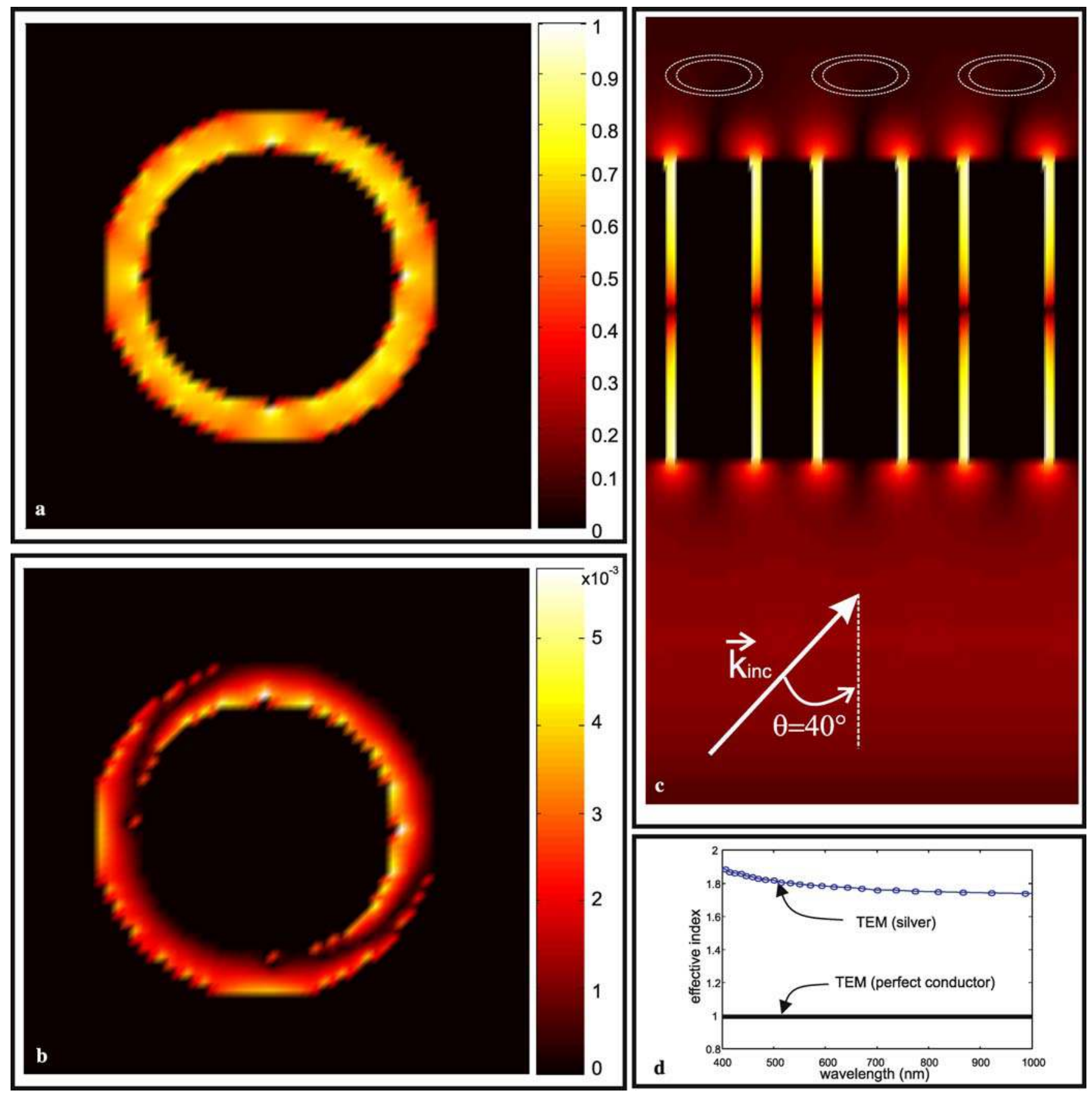

FIGURE 4 Modulus of the radial (a) and azimuthal (b) electric field components calculated at $100 \mathrm{~nm}$ from the exit side of the structure for parameters of point A in Fig. 3. These two quantities are normalized according to the maximum of the radial component. (c) Cross section in the $x z$ plane of the electric field amplitude in logarithmic scale. (d) Effective index of the TEM mode for an infinite coaxial waveguide made in perfect conductor or in silver. The silver dispersion is described by a Drude model $^{1}$ and the calculations [22] were done for $p=300 \mathrm{~nm}$

ble metals will play a positive role to bypass this constraint because the effective index of the TEM-like mode is larger than 1 (see Fig. 4d). This means that a phase-matching condition can be obtained with a thin metallic layer. We are

\footnotetext{
${ }^{1}$ The dispersion of silver is described by a Drude model that is expressed by $\varepsilon(\omega)=1-$ $\omega_{\mathrm{p}}^{2} /(\omega(\omega+\mathrm{i} \gamma))$ with $\omega_{\mathrm{p}}=1.374 \times 10^{16} \mathrm{rad} / \mathrm{s}$ and $\gamma=3.21 \times 10^{13} \mathrm{rad} / \mathrm{s}$.
}

working to integrate a dispersive model (Drude or Drude-Lorentz model) in our oblique-incidence FDTD algorithm to numerically expand the obtained results in the visible domain.

ACKNOWLEDGEMENTS This work is partially supported by the European Network of Excellence on Micro Optics (NEMO). The author is grateful to A. Belkhir for providing computational assistance.

\section{REFERENCES}

1 E. Yablonovitch, J. Opt. Soc. Am. B 10, 283 (1993)

2 J-M. Lourtioz, A de Lustrac, F. Gadot, S. Rawson, A. Chelnokov, T. Brillat, A. Ammouche, J. Danglot, O. Vanbesien, D. Lippens, J. Lightwave Technol. 17, 2025 (1999)

3 T.W. Ebbesen, H.J. Lezec, H.F. Ghaemi, T. Thio, P.A. Wolff, Nature 391, 667 (1998)

4 F.I. Baida, D. Van Labeke, Opt. Commun. 209, 17 (2002) 
5 M. Soljacic, J.D. Joannopoulos, Nat. Mater. 3, 211 (2004)

6 M. Roussey, F.I. Baida, M.-P. Bernal, J. Opt. Soc. Am. B 24, 1416 (2007)

7 H.J. Lezec, A. Degiron, E. Devaux, R.A. Linke, L. Martin-Moreno, F.J. Garcia-Vidal, T.W. Ebbesen, Science 297, 820 (2002)

8 F.I. Baida, D. Van Labeke, B. Guizal, Appl. Opt. 42, 6811 (2003)

9 U. Leonhardt, Science 312, 1777 (2006)

10 D. Schurig, J.J. Mock, B.J. Justice, S.A. Cummer, J.B. Pendry, A.F. Starr, D.R. Smith, Science 314, 977 (2006)
11 J.B. Pendry, Phys. Rev. Lett. 85, 3966 (2000)

12 C. Luo, S.G. Johnson, J.D. Joannopoulos, J.B. Pendry, Phys. Rev. B 65, 201104 (2002)

13 J.B. Pendry, D. Schurig, D.R. Smith, Science 312, 1780 (2006)

14 B.A. Munk, Frequency Selective Surfaces, Theory and Design (Wiley, New York, 2000)

15 E. Topsakal, J.L. Volakis, Antennas Wireless Propag. Lett. 3, 236 (2004)

16 D. Van Labeke, D. Gérard, B. Guizal, F.I. Baida, L. Li, Opt. Express 14, 11945 (2006)
17 T. Thio, Nat. Nanotechnol. 2, 136 (2007)

18 F.I. Baida, D. Van Labeke, G. Granet, A. Moreau, A. Belkhir, Appl. Phys. B 79, 1 (2004)

19 T. Grosjean, A. Sabac, D. Courjon, Opt. Commun. 252, 12 (2005)

20 A. Belkhir, F.I. Baida, J. Comput. Phys., submitted

21 P. Lalanne, J.P. Hugonin, S. Astilean, M. Palamaru, K.D. Möller, J. Opt. A Pure Appl. Opt. 2, 48 (2000)

22 F.I. Baida, A. Belkhir, D. Van Labeke, O. Lamrous, Phys. Rev. B 74, 205419 (2006) 